\title{
Addressing the Obsolescence of End-User Devices: Approaches from the Field of Sustainable HCI
}

\author{
Christian Remy and Elaine M. Huang \\ Department of Informatics, University of Zurich, Switzerland \\ \{remy, huang\}@ifi.uzh.ch
}

\begin{abstract}
The progress of technological development and the resulting rapid replacement of end-user devices has brought increasing issues of electronics waste upon our society. Interaction designers and researchers within the field of HumanComputer Interaction have begun to tackle issues of environmental sustainability in recent years, including the problem of obsolescence. By considering the experiential aspects of obsolescence and the ways in which interaction design could have an impact on experience, the field presents promising approaches with potential to contribute to and complement current materials-focused solutions. In this chapter, we report on a survey of sustainable human-computer interaction research that investigates or addresses issues of obsolescence, presenting challenges as well as opportunities for interaction designers to contribute to solving these issues.
\end{abstract}

Index Terms-Human-Computer Interaction, Sustainable HCI, Sustainable Interaction Design, Consumer Electronics, Obsolescence.

In recent years, the field of Sustainable Human-Computer Interaction (SHCI) evolved from an emerging topic to an established, well-published sub-community within the field of $\mathrm{HCI}$, appealing to a variety of environmental sustainability issues, among them the issue of technology obsolescence. We present an overview of SHCI literature that investigates or addresses issues of obsolescence by focusing on the implications for user experience and how influencing the user experience might change the pathway to obsolescence.

Our approach was to consider all publications related to both HCI and sustainability that address obsolescence directly or indirectly. In the literature review process, our focus was to gather insights through two primary approaches: first, identifying common themes in the solutions proposed for interaction design; second, highlighting challenges mentioned in SHCI research emerging from previous work. Three major themes emerged from our analysis - values in design, re-use and longevity - and for each of those themes we identified a number of design considerations that we discuss in light of their significance to issues of obsolescence and potential challenges in application. These design considerations, discussed in detail in the chapter, are as follows:
- Values in Design:

o Pleasure Engineering

o Achieving Heirloom Status

o Ensoulment

o Slow Design

○ New Luxury

- Re-Use:

- Transferability

o Repair

o Re-use of Materials

o Augmentation

- Longevity:

○ Longevity of Functionality

○ Intrinsic Motivation for Longevity

The obsolescence of end-user devices is an issue that concerns many research fields and needs to be tackled on many different levels. The domain of HCI research, due to its focus on user-centered design, can contribute to solutions by addressing obsolescence by considering the user experience, the interaction between the device and its owner, and the influence of these factors on ownership, use, and disposal. We highlighted a variety of design considerations that open up opportunities to engage in new efforts to overcome the rapid replacement of consumer electronics. The categorization of obsolescence-related SHCI research allows us to identify potential solutions to the problem of obsolescence that have been repeatedly found to hold promise. Furthermore, it serves as a design space that highlights under-explored areas that offer new directions for research.

Although some of the approaches described in this chapter offer illustrative examples of their application to product design, many of them have yet to be applied in real-world practice. Therefore, future research needs to investigate how these concepts can be applied to the design of end-user devices in practice. We believe that in order to be successful, a concerted effort that makes use of design knowledge accumulated in SHCI in cooperation with researchers from other domains and stakeholders in practice is necessary.

This contribution is based on the book chapter of the same authors in Hilty LM \& Aebischer B (Eds), ICT Innovations for Sustainability, Springer Series Advances in Intelligent Systems and Computing, 2014. The full text can be found in the book. 